\title{
Deciphering single- and multi-particle trapping dynamics under femtosecond pulsed excitation with simultaneous spatial and temporal resolution
}

\section{Anita Devi}

Indian Institute of Science Education and Research Mohali

\section{Sumit Yadav}

Indian Institute of Science Education and Research Mohali

Arijit De ( $\square$ akde@iisermohali.ac.in )

Indian Institute of Science Education and Research Mohali

\section{Research Article}

Keywords:

Posted Date: January 13th, 2022

DOI: https://doi.org/10.21203/rs.3.rs-1238666/v1

License: (c) (i) This work is licensed under a Creative Commons Attribution 4.0 International License.

Read Full License

Version of Record: A version of this preprint was published at Scientific Reports on March 30th, 2022. See the published version at https://doi.org/10.1038/s41598-022-09251-4. 


\section{Abstract}

Recent theoretical and experimental studies have shed light on how optical trapping dynamics under femtosecond pulsed excitation are fine-tuned by optical and thermal nonlinearities. Here, we present experimental results of nonlinear optical trapping of single and multiple polystyrene beads (of $1 \mu \mathrm{m}$ diameter). We show how integration and synchronization of bright-filed video microscopy with confocal detection of backscatter provide both spatial and temporal resolution required to capture intricate details of trapping dynamics. Such spatiotemporal detection is promising to have far-reaching applications in exploring controlled optical trapping and manipulations harnessed by optical and thermal nonlinearities.

\section{Main Text}

Optical tweezer is a non-invasive tool that is used for the contact-free manipulation of small objects by harnessing the high-intensity gradient of a tightly focused laser beam [1]. Over nearly five decades, it revolutionized the exploration of microscopic world with far-reaching applications across disciplines [2]. For trapping of particles with a wide range of sizes, mostly continuous-wave (CW) lasers were used with a few notable exceptions where femtosecond lasers were used [3-6] which reported that micrometer-sized silica or polystyrene particles show similar (at less power) [3-4] or less (at high power) [5-6] trap stiffness compared with $\mathrm{CW}$ excitation. In a quest of understanding the fundamental physics underlying these puzzling observations, optical and thermal nonlinearity was demonstrated to modulate the trapping force under femtosecond pulsed excitation both theoretically as well as experimentally [7-11]. As delineated in these works focused on optical trapping dynamics under pulsed excitation, at low average power, nonlinear effects are insignificant, hence, trap stiffness is equivalent to CW excitation. However, at high average power, nonlinear optical effects are significant which results in more asymmetry in the potential well; while the trapping potential well become steeper before the geometric trap center, it becomes shallower on the other side; equivalently saying, the potential barrier to come out of the trapping well or the 'escape potential' decreases. Consequently, trap stiffness is less than $\mathrm{CW}$ excitation. In addition to mapping this escape potential, experiments reveled a quick 'adjustment' of the initially dragged particle characterized by a movement in forward direction which is a result of laser-induced thermal effects in the presence of the particle $[10,11]$.

One crucial aspect to disentangle the intricate events in these experiments [10-11] was to integrate various detection methods (for example, two-photon fluorescence and backscatter detection) using a multimodal optical tweezers set-up [12] since different methods furnish complementary information. However, in addition to integration, synchronization of different detection modalities is often needed for a complete understanding of trapping dynamics which we present here. In this letter, we demonstrate how simultaneous detection of bright-field video microscopy provide a complete understanding of optical trapping dynamics of single and multiple particles inside a nonlinear laser trap created by a femtosecond pulse-train. 
In Figure 1, we show the optical and optomechanical components of the bench-top femtosecond optical tweezer. A detailed discussion of construction and calibration of this apparatus may be found elsewhere [12]. Briefly, following spatial mode clean-up, the expanded laser beam from a Ti-Sapphire oscillator, producing pulses spectrally centered on $\sim 800 \mathrm{~nm}$ at a repetition-rate of $80 \mathrm{MHz}$, is used to fill the back aperture of a microscope objective which is used to trap the particles as well as to collect the back-scatter as well as the transmitted white light. Diluted and sonicated suspension of $1 \mu \mathrm{m}$ diameter polystyrene beads in deionized water is put onto a No 1 glass coverslip (of $100 \mu \mathrm{m}$ thickness) and the position of the focal plane from the coverslip surface is kept at $\sim 50 \mu \mathrm{m}$ to avoid any interaction between the particles and glass surface. The transmitted white light and a small fraction $(<5 \%)$ of the backscatter pass through a dichroic; further using a 10:90 (R:T) beam-splitter, the scatter and the white light are detected separately and simultaneously. The transmitted white light was imaged onto the CMOS sensor of a camera for video microscopy at a rate of 150 frames-per-second (in 'cropped' mode). The reflected scatter was routed to a confocal aperture (using a $200 \mu \mathrm{m}$ pinhole and an IR filter) for point detection of back-scatter signal using a PMT, recorded by an automated oscilloscope at $400 \mu$ s time interval and the data is plotted after 10-point moving averaging of raw data; however, data analysis is done using the raw data (to exclude artifacts associated with averaging [13]) and trap stiffness values are calculated by averaging over more than 10 sets of data.

Although, the sensitivity of bright-field video microscopy on lateral position and that of confocal detection of backscatter on axial position may not seem to complement each other; however, it should be brought in mind that any movement away from the trap center renders more room to the particle(s) for lateral movement as well because of the converging/diverging trapping beam across the geometric focus. Thus the fluctuations in motions along $\mathrm{x}-\mathrm{y}$ - and $\mathrm{z}$ - directions are coupled and provide complementary spatial and temporal resolution, respectively, of the same observables.

In section S1 in the Supplementary Information (SI), we provide a detailed discussion on single-particle dynamics. Earlier, the single-particle dynamics of fluorescent dye coated particle was shown to proceed through sequential steps inside a nonlinear laser trap [10]. These steps are further confirmed here for the uncoated particles. The time constants are found to be similar for both types of particles as both particles are the same in size and were trapped under similar experimental conditions. Interestingly, the confinement time (the total time over which the particle stays inside the trap, from drag to ejection) for a coated particle is less as compared to an uncoated particle in similar conditions. This is most likely due to destabilization of the trap following absorption-induced heating (i.e. downconversion of photons absorbed by the fluroresent dye molecules, via two-photon absorption, to heat); a detailed discussion and a comparison table shown in SI.

Figure 2a shows the back-scatter signal for two particles confined in the optical trap. The sudden rise in the back-scatter signal (step no. 3) indicates the dragging of second particle. Noticeably, unlike the singleparticle case, this rise is not followed by any drop in the signal that due to adjustment dynamics ([10], section $\mathrm{S} 1$ in $\mathrm{SI}$ ) because the shift in the equilibrium position (due to thermal nonlinearity) is not significant when second particle is dragged. This is because trapping potential is already modulated by 
the presence of first particle. So, further modulation due to dragging of second particle is not evident. The integrated signal from both the particles shows fluctuations which corresponds to the lateral shift of the second particle w.r.t the first particle. The confinement time of one particle in absence/presence of the second particle is quit different. Due to reduced accessible volume, the second particle do not exhibit any adjusment dynamics after initial drag. Eventually, the ejection dynamics for two particles, can have two possibilities: either both the particles leave the trap (marked as step no. 4 in figure 2a) or one particle leaves the trap earlier while another particle stays back. Figure $2 \mathrm{~b}$ shows the corresponding transmitted wide-field microscopic images of dragging of second particle (which can be dragged from any direction) and ejection of both the particles (which is always along axial direction). The transmitted images give the spatial resolution and by tracking the position of particle(s) in the trap, we get the position distribution and the trajectory of particle. Figures $2 \mathrm{c}-\mathrm{d}$ show the position distribution of particle(s) along $\mathrm{x}$ - and $\mathrm{y}$-axis and figure $2 e$ shows the trajectory of particles inside the nonlinear optical trap. The trajectory of single particle shows a clear shift in the mean position on the drag of second particle. A shift in the mean position of the particle's trajectories is observed (from $x=-21.24 n m, y=5.67 n m$ to $x=5.59 n m, y=-1.47$ $\mathrm{nm}$ ) when the second particle is trapped. However, when two particles are present within the optical trap, fluctuations in the position increase rapidly due to the collision between the particles. Since the particles adjust themseleves along axial direction in addition to lateral direction within the trap and stays longer time inside the trap at low average power $(14.10 \mathrm{~mW})$, deconvoluting their mean position from video micrographs (which is a collection of -xy projection on CMOS plane) is quit challenging. Also, the position distribution along $\mathrm{x}$ - and $\mathrm{y}$-axis for two particles dynamics is also merged in such a way that it becomes difficult to disentangle which can be seen from the figures $2 \mathrm{c}-\mathrm{e}$. Hence, a clear distribution was not obtained along both $x$ - and $y$-axis. However, at high average power $(23.50 \mathrm{~mW})$, a clear shift in the particles' position distribution and distinct localization of individual particles are observed which can be seen from figures $2 \mathrm{f}-\mathrm{h}$. Because at high average power, escape potential decreases; consequently, the trap is more asymmetrical in nature for which particles' confinement time drops from 505 down to $84 \mathrm{sec}$. Besides, at high average power, potential well broadens, allowing particles to fluctuate more, resulting in a substantial shift in the mean position of the particle trajectory. Therefore, the two particles explore different regions in space but for a short time. Thus, we see two distinct spatial distribution in the trajectory of the particles as shown by a double-sided black arrow in figure $2 \mathrm{~h}$.

Figure 3 shows that the time scale of dragging and ejection is in the range of milliseconds and these timescales are further reduced with an increase in power. According to the Nyquist-Shannon sampling theorem, we need resolution in the hundreds of microseconds to identify this scale. Without a high temporal resolution, establishing the drag and ejection time for the second particle is quite difficult; Capturing images at a high frame rate with a standard CMOS camera and interpreting the data is quite challenging. Therefore, we employ a simultaneous point detection approach with a high temporal resolution to capture such fast dynamics [10].

In section $\mathrm{S} 2$ in the $\mathrm{SI}$, we provide a detailed discussion on dependence of drag and ejection times on laser power. The drag times are different for both the first and second particle as the potential well is different for both the particles. The drag for second particle depends on the modulated potential created 
by the first particle and the drag time for second particle is more than the first particle at low average power $(\sim 4.7 \mathrm{~mW})$, which changes with power. This might be due to a significant nonlinear effect at a high power level. The ejection of both the particles is fast as compare to single-particle ejection as two particles posses high collisional energy.

Figure 4a shows the back-scatter signal (from point detection) from more than two particles being trapped inside the nonlinear optical trap. A sudden rise of the signal indicates the drag of third particle (marked by no. 4) as shown in the zoomed-in trace(the first red circle) in figure 4a. The decay in the signal followed by sudden rise indicates the ejection of one of the residing particles, (not the adjustment). For the particle size and conditions (wavelength of trapping beam, NA of objective, etc) used in our experiment, no more than two particles can reside in the trap. The zoomed-in trace (the second red circle) in figure 4 a shows the ejection of both the particles (marked by no. 5). The corresponding transmitted wide-field microscopy images are shown in figure $4 \mathrm{~b}$. Here, yellow dotted circle represents the trap center, the red arrow shows the dragging direction of second and third particles. From these images, it is very clear that when third particle is dragged, one of the existing particles has to leave the trap which is marked by a blue circle. The ejecting particle is not visualised clearly because ejection is along the axial direction. Figures $4 c-d$ show the plots of $x$ - and $y$-position of the particles against time, and a shift in the mean position of the particles (a diagonal shift most likely, although this shift can be in any direction) is observed when second or third particles are dragged within the focal volume. The dragging of third and ejection of one of the existing particles are marked by a red circle. Figure $4 \mathrm{e}$ shows the trajectories of the particles here, blue represents the trajectory of single particle, green represents the trajectory after the drag of second particle, and red represents the trajectory after the drag of third particle and ejection of the existing one. The shift in mean positions of the particle trajectories are also mentioned. Similar, behavior is observed at different average powers as well.

To conclude, we have shown how synchronized spatiotemporal detection provides a vivid picture of optical trapping dynamics of single and multiple particles under femtosecond pulsed excitation.

\section{Declarations}

\section{Acknowledgements:}

This research was supported by Early Career Research Award from SERB, DST (Grant No:

ECR/2016/000467) and by Start-up Grant from IISER Mohali. AD and SY acknowledge IISER Mohali and UGC, respectively, for Graduate Fellowships.

\section{Additional information:}

The authors declare no competing financial interests.

$A D$ and SY contributed equally to this work supervised by AKD. All authors participated in writing the manuscript reviewed it. 


\section{Data availability:}

The data that support the findings of this study are available within the article and its supplementary material.

\section{References}

1. A. Ashkin, J. M. Dziedzic, J. E. Bjorkholm, and S. Chu, "Observation of a single-beam gradient force optical trap for dielectric particles," Opt. Lett. 11(5), 288-290 (1986).

2. A. Ashkin, "Optical trapping and manipulation of neutral particles using lasers: A reprint volume with commentaries," World Scientific (2006).

3. B. Agate, C. T. A. Brown, W. Sibbett, and K. Dholakia, "Femtosecond optical tweezers for in-situ control of two-photon fluorescence," Opt. Express 12, 3011-3017 (2004).

4. J. C. Shane, M. Mazilu, W. M. Lee, and K. Dholakia, "Effect of pulse temporal shape on optical trapping and impulse transfer using ultrashort pulsed lasers," Opt. Express 18, 7554-7568 (2010).

5. T-H Liu, W-Y. Chiang, A. Usman, and H. Masuhara, "Optical trapping dynamics of a single polystyrene sphere: Continuous wave versus femtosecond lasers," J. Phys. Chem. C 120, 2392-2399 (2016).

6. W. Y. Chiang, A. Usman, and H. Masuhara, "Femtosecond pulse width dependent trapping and directional ejection dynamics of dielectric nanoparticles," J. Phys. Chem. C 117, 19182-19188 (2013).

7. A. Devi, and A. K. De, "Theoretical investigation on nonlinear optical effect in laser trapping of dielectric nanoparticles with ultrafast pulsed excitation," Optics Express 24, 21485-21496 (2016).

8. A. Devi, and A. K. De, "Generalized description of the nonlinear optical force in laser trapping of dielectric nanoparticles," Phys. Rev. Res. 2, 043378 (2020).

9. A. Devi, and A. K. De, "A unified treatment of nonlinear optical force in laser trapping of dielectric particles of varying sizes," Phys. Rev. Res. 3 (3), 033074 (1-21), (2021).

10. A. Devi, S. Yadav, and A. K. De, "Dynamics of a dielectric microsphere inside a nonlinear laser trap," Appl. Phys. Lett. 117 (16), 161102 (1-6) (2020).

11. A. Devi, S. Yadav, and A. K. De, "Complementing two-photon fluorescence with back-scatter detection to decipher laser trapping dynamics," Opt. Lett. (Under review).

12. A. Devi, and A. K. De, "A table-top compact multimodal nonlinear laser tweezer," Opt. Commun. 482, 126440 (2020).

13. A. Devi, and A. K. De, "Simultaneous detection of two-photon fluorescence and back-scattered signal of optical trapping of dielectric nanoparticles under femtosecond pulsed excitation," J. Nanophotonics 13(2), 020501(1-8) (2019).

\section{Figures}




\section{Spatial-resolution}

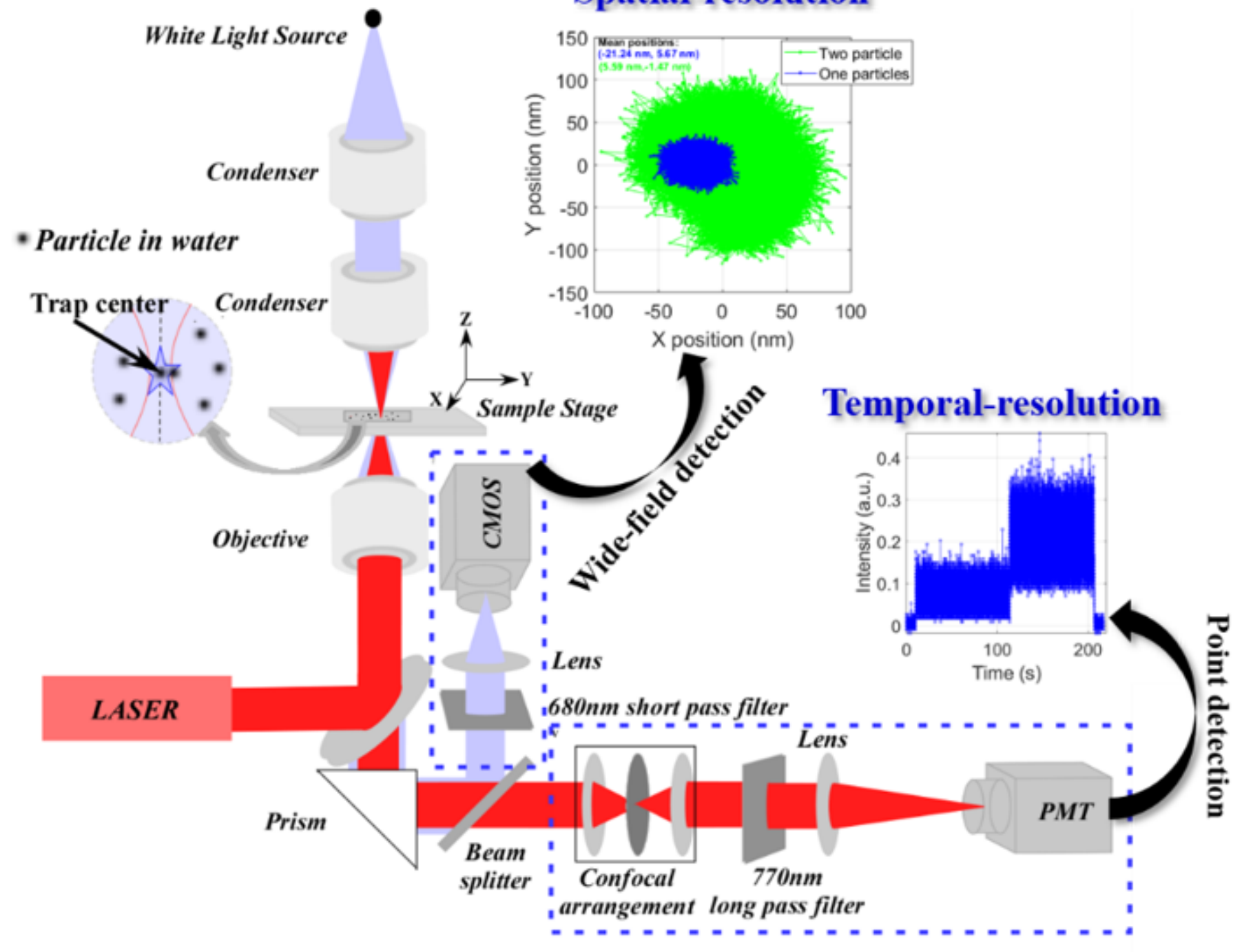

Figure 1

Schematic of the experimental setup. 

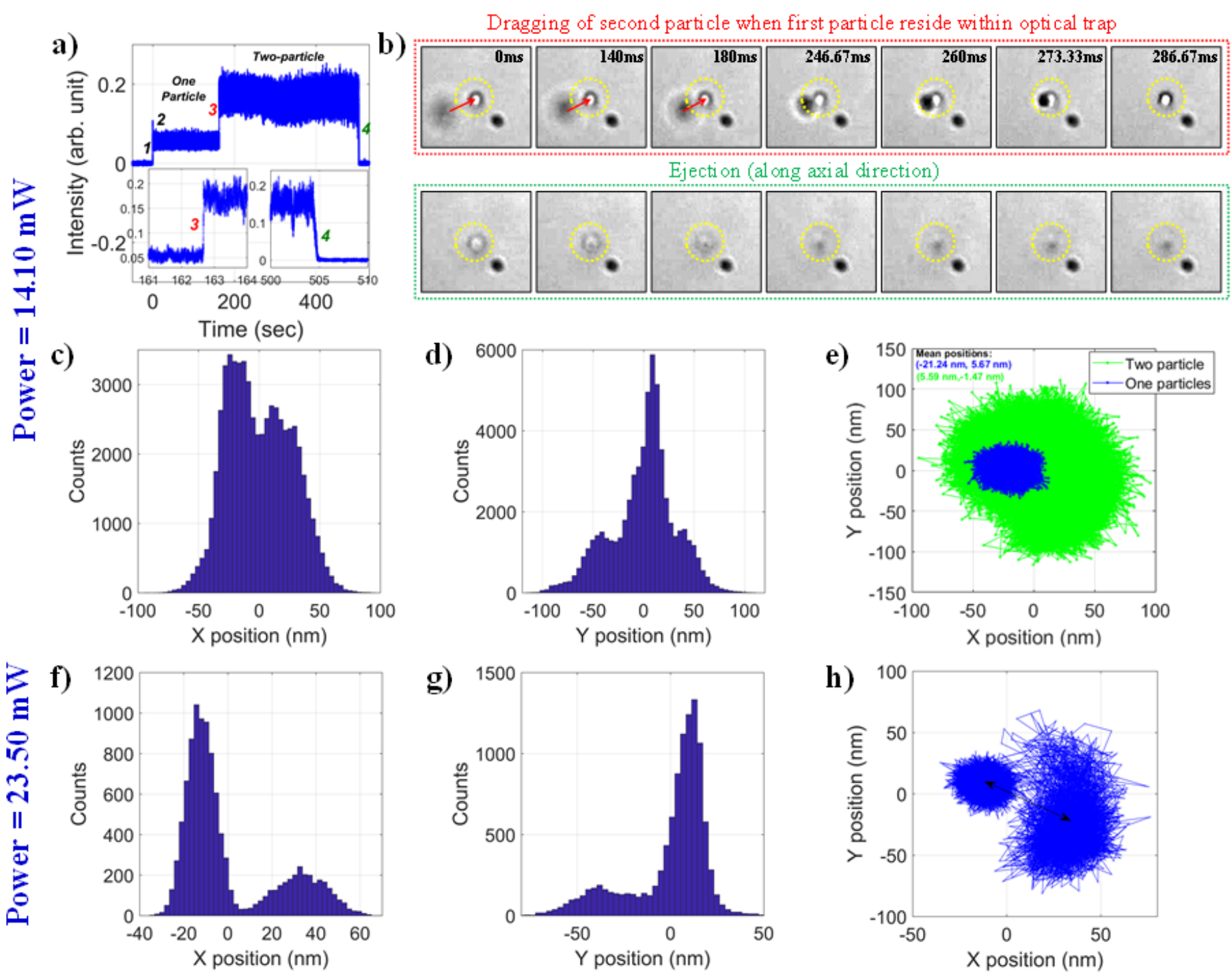

Figure 2

a) Back-scatter signal for two particles, b) transmitted wide-field microscopy images of the particles to map dragging of latter particle when first particle is residing inside the nonlinear optical trap and ejection of both the particles; position distribution along c) x-axis, d) y-axis, e) $x$-y trajectory; and f) $x$-axis, g) yaxis, h) $x$-y trajectory. The data are for confinement of two particles within the nonlinear optical trap at $14.10 \mathrm{~mW}$ (first and second row), and $23.50 \mathrm{~mW}$ (third row) average power under pulsed excitation. 

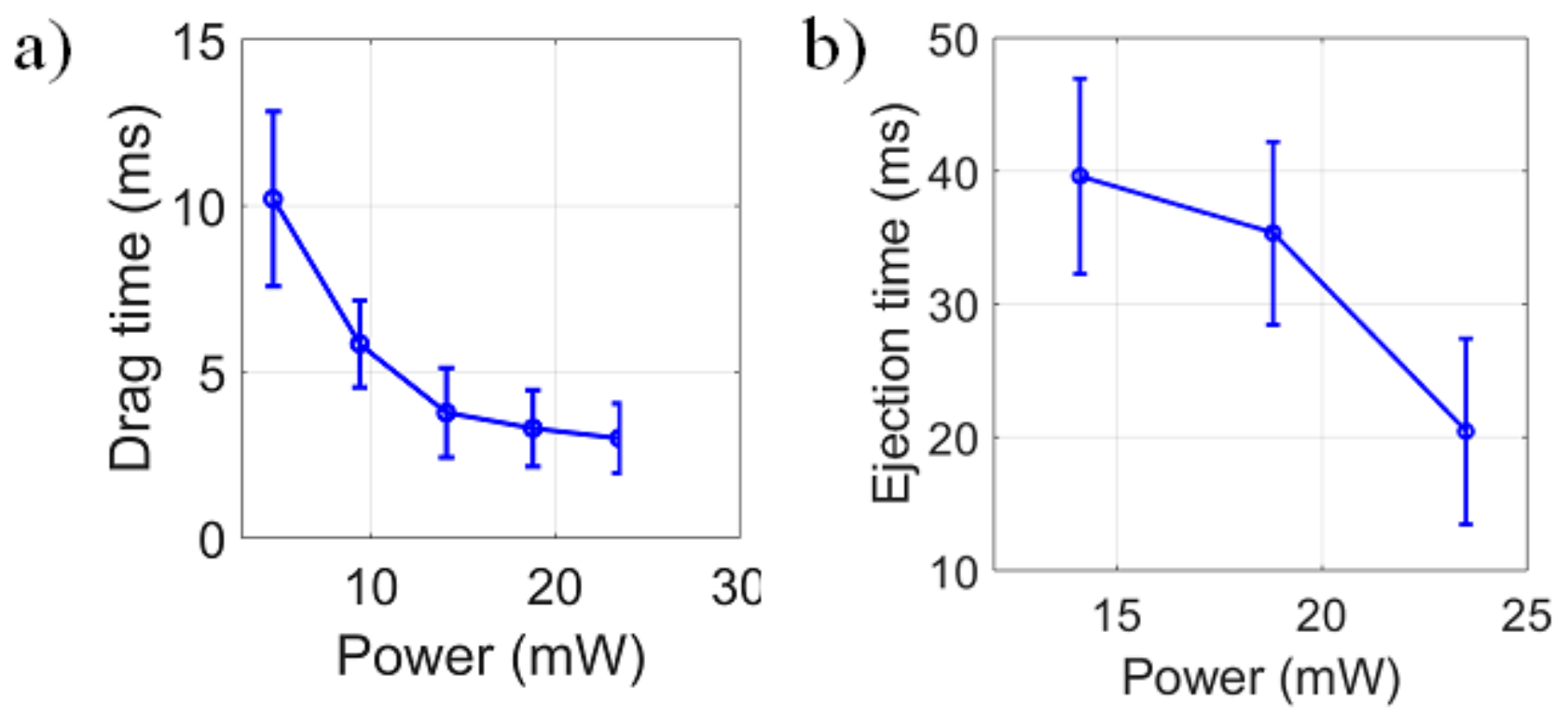

Figure 3

a) Drag, and b) ejection times for the second particle against average power under pulsed excitation.

a)

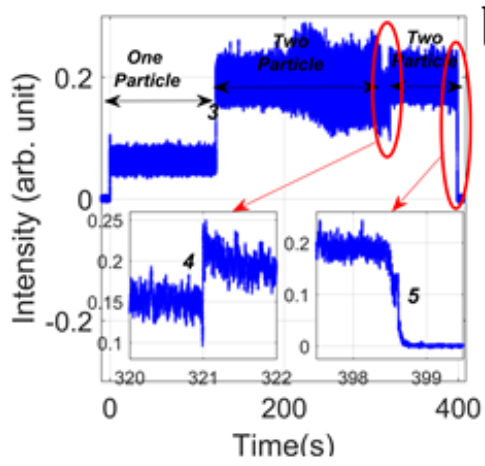

c)

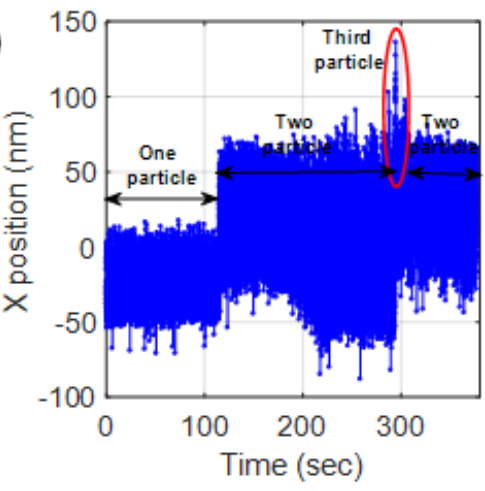

b)

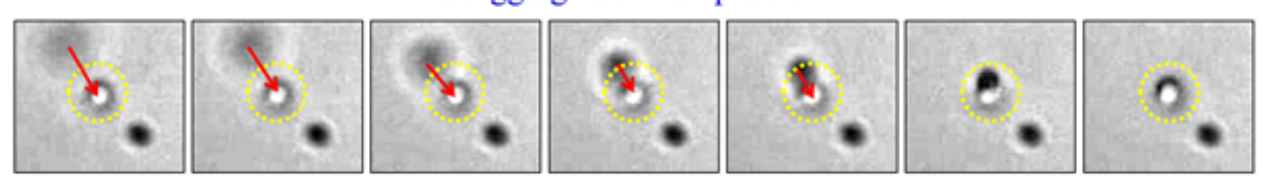

Dragging of third particle and ejection of one of the existing particles

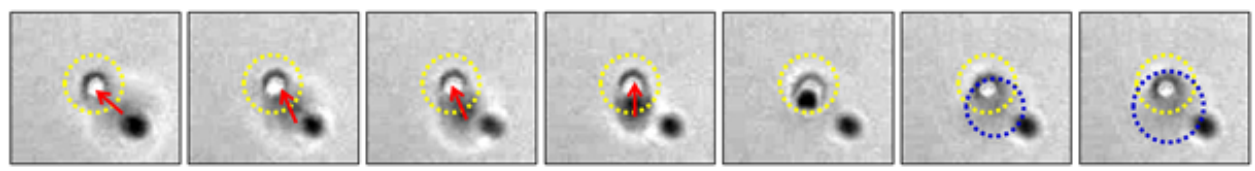

d)

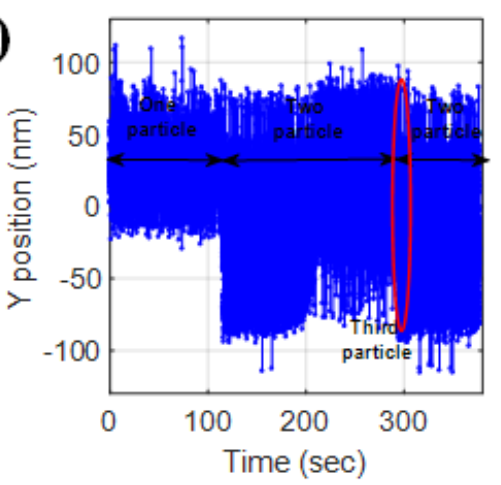

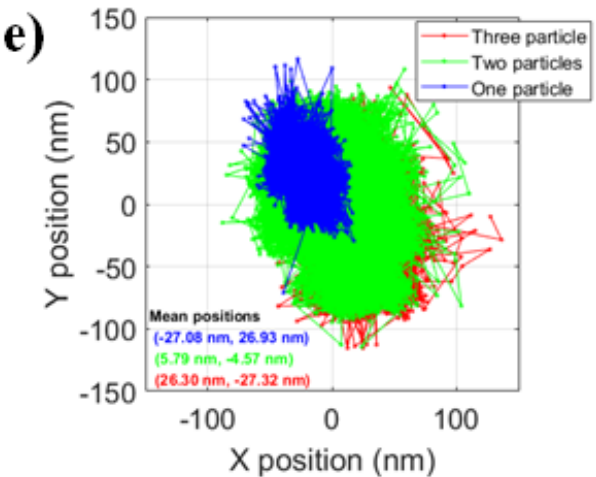

Figure 4 
a) Back-scatter signal for multiple (more than two) particles, b) transmitted wide-field microscopy images of the particles to map dragging of second and third particle when first and among one of two particles is residing inside the nonlinear optical trap respectively. Position along c) $x$-axis, d) $y$-axis against time, and e) $x-y$ trajectory. The data are for confinement of multiple particle within the nonlinear optical trap at $14.10 \mathrm{~mW}$ average power under pulsed excitation.

\section{Supplementary Files}

This is a list of supplementary files associated with this preprint. Click to download.

- SciRepSI.pdf 